\title{
Structural and predictive properties of the Emotional Quotient Inventory Youth Version - Short Form (EQ-i:YV[S])
}

\begin{abstract}
Emotional Intelligence (EI) is a popular construct with concentrated areas of application in education and health contexts. There is a need for reliable and valid measurement of EI in young people, with brief yet sensitive measures of the construct preferable for use in time-limited settings. However, the proliferation of EI measures has often outpaced rigorous psychometric evaluation (Gignac, 2009). Using data from 849 adolescents (407 females; 422 males) aged 11 to 16 years (mean age 13.4; SD = 1.2 years), the current paper systematically examined the structural and predictive properties of a frequently employed measure of adolescent trait EI - the Emotional Quotient Inventory Youth Version Short Form - EQ-i:YV(S) (Bar-On \& Parker, 2000). Whilst the intended multidimensional factor structure was recovered through confirmatory factor analysis, the statistical and conceptual coherency of the underlying model was inadequate. Using a multi-trait multi-method approach, the EQ-i:YV(S) was found to converge with other measures of EI, however, evidence for divergent validity (Big Five personality dimensions) was less robust. Predictive utility for adolescent mental health outcomes (depression; disruptive behavior) was also limited. Findings suggest that use of the EQi:YV(S) for predictive or evaluative purposes should be avoided until refinements to the scale are made.
\end{abstract}

Keywords: emotional intelligence; Emotional Quotient Inventory Youth Version Short Form; confirmatory factor analysis; reliability; validity; multi-trait multi-method 
PSYCHOMETRIC PROPERTIES: EQ-I:YVS

Emotional intelligence (EI) is an individual differences construct which indexes how well we perceive, communicate, regulate and understand our own emotions and the emotions of others (Zeidner, Matthews, \& Roberts, 2009). The construct can be measured in two ways: either as an ability or trait. Proponents of the ability perspective (hereafter AEI) view EI as a distinct form of intelligence for reasoning about emotion, represented by a set of interactive cognitive abilities specialized for complex emotional information processing (Mayer, Roberts, \& Barsade, 2008). These skills (perceiving, using, understanding, managing emotion) can be measured veridically via tests of maximal performance, akin to traditional cognitive testing. In contrast, the trait approach (hereafter TEI) is concerned with self-perceived emotional functioning, preferences and qualities, evidenced through traits such as empathy, adaptability, emotional expression and assertiveness. A wide array of TEI models/measures exist (see Perez, Petrides, \& Furnham, 2005) yet all share a common self-report approach to measurement, evaluating typical performance (i.e., perceptions of attributes via internal appraisal) in the same way as traditional personality testing.

With links to a range of adaptive outcomes including emotional/physical health, personal/social competence, and occupational success (Brackett, Rivers, \& Salovey, 2011; Martins, Ramalho, \& Morin, 2010; Van Rooy \& Viswesvaran, 2004) together with evidence that EI can be trained or honed through intervention programmes (Durlak, Weissberg, Dymnicki, Taylor, \& Schellinger, 2011), interest in EI shows no sign of abating. This has led to a proliferation of tools designed to tap emotionally intelligent behavior, many of which have not undergone (or do not meet requirements of) full psychometric validation, leaving the field open to criticism (Brody, 2004; Wilhelm, 2005). For instance, the notion that EI may be unable to 
PSYCHOMETRIC PROPERTIES: EQ-I:YVS

offer anything new to the prediction of adaptive outcomes beyond allied variables, such as personality, has been a long-standing point of contention (Brody, 2004; Schulte, Ree, \& Carretta, 2004). With attention now turning to youth-based research in EI, systematic examination and validation of available measurement instruments is required (Gignac, 2009). The current paper aids in this endeavor by exploring the psychometric properties of one such measure - the Emotional Quotient Inventory Youth Version -Short Form (Bar-On \& Parker, 2000).

\section{Why measure EI in young people?}

Evidence suggests that young people with higher levels of EI experience fewer depressive, anxious, and somatic symptoms; exhibit less disruptive behavior and attention deficit hyperactivity disorder symptomatology (Kristensen et al., 2014; Mavroveli, Petrides, Rieffe, \& Bakker, 2007; Rivers et al., 2012; Williams, Daley, Burnside, \& Hammond-Rowley, 2009); achieve greater exam success (Perera \& DiGiacomo, 2013); and display pro-social behavior (Mavroveli \& Sánchez-Ruiz, 2011). Researchers are now engaged in deconstructing when and how A/TEI exerts a beneficial effect in young people (e.g., Davis \& Humphrey, 2014; Qualter, Gardner, Pope, Hutchinson, \& Whiteley, 2012) to ascertain whether EI is truly 'adaptive' and provide support for the implementation of school-based social and emotional learning programmes (e.g., Brackett, Rivers, Reyes, \& Salovey, 2012). Pivotal to the advancement of such applied research is the development of reliable and valid measures of EI suitable for use with children and adolescents. As this typically necessitates battery testing of large samples of young people in time-limited settings (e.g., school classrooms), a brief yet sufficiently broad measure (which fully captures all aspects of EI i.e. emotion perception, management, use and understanding) is optimal. This would also be desirable for more targeted research in clinical settings, where rapid screening of individuals might be required and/or the cognitive demands 
PSYCHOMETRIC PROPERTIES: EQ-I:YVS

imposed by lengthy test administration are too great. Additionally, measures of EI should meet basic psychometric standards concerning reliability (e.g., test-retest reliability; internally consistent test items) and validity (e.g., robust factor structure; convergence/divergence from key individual difference constructs; predict meaningful criteria with unique capacity) (Gignac, 2009). As shall be seen below, there are presently few measures available for use with young people, which meet these criteria.

\section{Measurement of EI in youth}

For AEI, a youth version of the Mayer-Salovey-Caruso Emotional Intelligence TestYouth Version (MSCEIT-YVR) suitable for 10-17 year olds, is currently under validation (Papadogiannis, Logan, \& Sitarenios, 2009). The tool indexes capacity to perceive, use, understand, and manage emotions via a series of multiple choice and 'rate the extent' questions scored according to expert ratings. One hundred and one items take about an hour to complete (depending upon respondent age) which limits its use with large groups in applied settings. Presently, the MSCEIT-YVR is the only omnibus measure of child/adolescent AEI. The Situational Test of Emotion Management for Youth (STEM-Y see MacCann, Wang, Matthews, \& Roberts, 2010) has shown adequate internal consistency to provide a brief indicator of skill in emotion regulation in 11-15 year olds. However, the breadth of the instrument is limited given that only one discrete component of the AEI sampling domain is covered; researchers would need to employ additional 'non-AEI’ instruments to measure allied AEI skills (see e.g.,Williams et al., 2009).

A greater range of self-report style TEI measures for youth exist, however these are limited by psychometric shortcomings and/or their capacity to offer brief yet sufficiently broad measures of EI. The Schutte Self-Report Emotional Intelligence Test (SREIT) (Schutte et al., 
PSYCHOMETRIC PROPERTIES: EQ-I:YVS

1998) is limited by inadequate sub-scale reliabilities and an inconsistent factor structure (Saklofske, Austin, \& Minski, 2003) meaning analyses are restricted to use of a total/global TEI score and do not permit scrutiny of performance across the full range of EI competencies. The same issue applies to the use of the Trait Emotional Intelligence Questionnaire-Adolescent short form (TEIQue-ASF) (Petrides, 2009) despite demonstrating good reliability and predictive validity (e.g., Davis \& Humphrey, 2012). However, the 30-item Emotional Quotient Inventory: Youth Version Short Form (EQ-i-YV (S)) (Bar-On \& Parker, 2000) offers a multidimensional alternative to the SREIT and TEIQue-ASF which can be completed in less than 15 minutes by 718 year olds. Developed from the long-form youth measure (EQ-i:YV - 60 items), the EQi:YV(S) taps self-perceived intrapersonal competencies (emotional self-awareness; self-regard; assertiveness; self-actualization; independence), interpersonal proficiency (empathy; relationships; social responsibility), stress management (impulse control; stress tolerance) and adaptability (flexibility; problem-solving; reality testing). A positive impression scale is also included to identify socially desirable responding. Consequently, 5 scale scores together with a total 'EQ' score can be derived. With the inclusion of an array of personal competencies, traits, and qualities beyond the 'traditional' facets of EI (i.e., perception, understanding, management and use of emotional cues), the EQ-i:YV/YV(S) has been found to predict a range of adolescent outcomes including addiction related behavior (Parker, Summerfeldt, Taylor, Kloosterman, \& Keefer, 2013), ADHD symptomatology (Kristensen et al., 2014), psychopathology (Wong \& Ang, 2007) and academic achievement (Qualter et al., 2012). However, to the authors’ knowledge, no studies have yet examined whether predictive associations hold in the presence of conceptually related constructs such as personality. The distinctiveness of EQ-i:YV(S) scores warrants close attention given the scale’s broadband coverage of TEI (Zeidner et al., 2009). 
PSYCHOMETRIC PROPERTIES: EQ-I:YVS

Indeed, beyond normative data ( $\mathrm{N}=9172$, Bar-On \& Parker, 2000) which suggests the EQ-i:YV(S) possesses satisfactory internal reliability (.65 - .87) and 3-week test re-test reliability (.81 [interpersonal] - .88 [stress management]), there has been no systematic psychometric scrutiny of the tool. Moreover, it is notable that this initial validation data was drawn from the EQ-i:YV data pool (as the 'parent' measure from which the EQ-i:YV(S) was developed). As has been noted in the literature (Smith, McCarthy, \& Anderson, 2000) this practice is insufficient for credible short-form psychometric analysis; the reduced length and content coverage of the EQ$\mathrm{i}: \mathrm{YV}(\mathrm{S})$ make it a standalone measure of TEI which requires independent validation. In particular, it must be shown that the multifactorial nature of the tool is preserved in the abbreviated form. This has received little attention from researchers to date. Whilst Parker et al., (2005) recovered a correlated, four-factor structure (intrapersonal, interpersonal, adaptability, stress management) in long-form data from North American children/adolescents, two items exhibited very low factor loadings on the 'intrapersonal' dimension and there was no consideration of the comparative fit of alternative factor structures (e.g., unidimensional; secondorder model). Kun et al., (2012) examined the factor structure of the short-form in Hungarian adolescents, however, the inclusion of the 'positive impression' index, removal of 6 items (notably from the inter/intrapersonal dimensions), and lack of multi-model testing, limits the generalizability of their final factor solution. It is also notable that a recent CFA of a significantly abbreviated 12-item version of the EQ-i:YV (EQ-i:YV-Brief) found stress management to be orthogonal to the other three EQ-i dimensions (Keefer, Holden, \& Parker, 2013). Given its derivation from (EQ-i:YV) and dilution to (EQ-i:YV-Brief) related forms, this evidence suggests scrutiny of the factorial validity of the EQ-i:YV(S) is urgently required.

\section{The current study}


PSYCHOMETRIC PROPERTIES: EQ-I:YVS

Despite popular use within educational and clinical domains (Humphrey et al., 2011), there has been little systematic analysis of the psychometric properties of the EQ-i youth forms. Consequently, this study aims to provide preliminary psychometric data for the EQ-i:YV(S) drawn from a large sample of UK adolescents. The only existing structural analysis of the tool is limited (Kun et al., 2012), with examinations of the long and brief versions of the EQ-i:YV suggesting potential issues with the factor structure (Keefer et al., 2013; Parker, Saklofske, Shaughnessy, et al., 2005). The current study will scrutinize the validity of the measure through a series of confirmatory factor analyses to address a) whether the multifactorial nature of the tool is robust (i.e., TEI is best represented as four distinct, yet related subscales vs. a unidimensional, global TEI with/without four subfactors) and b) whether this structure holds across males and females (measurement invariance). The latter issue has not yet been examined in EQ-i:YV(S) data and is important given the commonplace reporting of sex differences in TEI - usually finding a female advantage (e.g., Mavroveli \& Sánchez-Ruiz, 2011; Qualter et al., 2012) - and the norming of sex-specific EQ-i:YV(S) scores (Bar-On \& Parker, 2000).

Additionally, this will be the first study to systematically examine the convergent, divergent and predictive validity of the EQ-i:YV(S) in relation to key mental health outcomes and allied constructs. EQ-i:YV(S) scores are expected to converge with scores from two alternative measures of EI, one self-report (TEIQue-ASF) and the other performance-based (MSCEIT-YV(R)) (i.e., moderate to robust correlation coefficients). Scores indexing broadband personality dimensions (i.e, Neuroticism, Extraversion, Openness, Agreeableness, Conscientiousness), are expected to diverge from EQ-i:YV(S) data (i.e., negligible to moderate associations). Following recommendations in the literature (Gignac, 2009; Parker, Keefer, \& Wood, 2011), analyses will follow a multitrait-multimethod latent variable modeling approach to 
PSYCHOMETRIC PROPERTIES: EQ-I:YVS

control for common method variance and measurement error - a statistical approach that has not yet been performed with EQ-i:YV(S) data. Scores should also significantly predict key mental health criterion (depression; disruptive behavior) over and above the influence of personality dimensions in order to disconfirm conceptual redundancy (Schulte et al., 2004) and lend credence to EI-based interventions with young people. This study will be the first to assess the incremental validity of EQ-i:YV(S) scores to predict key mental health criterion.

\section{Method}

\section{Participants}

Cross-sectional data were obtained from 849 adolescents (407 females; 422 males) aged 11 to 16 years $(M=13$ years, 4 months $S D=1.2$ years $)$ who attended one of seven participating schools located across the West Midlands and North Yorkshire, UK. Schools were selected via opportunity sampling, with study participation contingent upon parental consent and student assent. Socio-demographic data were consistent with national averages (Department for Education, 2011) e.g., 15.5\% vs. 15.9\% eligibility to claim free school meals with a diversity of ethnic backgrounds; 38.2\% White/White European, 36.7\% Asian/Asian British, 9.1\% Black/Black British, 4.1\% mixed background, 0.9\% Chinese and 1.3\% belonged to other ethnic groups (9.7\% missing or refusing to provide information).

\section{Materials \& Procedure}

Students who consented to participate were given verbal and written instructions and completed each measure (counterbalanced) within the whole-class setting (1 hour).

The Mayer-Salovey-Caruso Emotional Intelligence Test-Youth Version (MSCEIT:YV): Research Edition (Mayer, Salovey, \& Caruso, in press) is an AEI measure comprising 101 items tapping skill in perception, use, understanding and management of emotion. To index emotion 
PSYCHOMETRIC PROPERTIES: EQ-I:YVS

perception, participants rate a series of faces for emotional content using a 5-point scale; 1 (none at all) through to 5 (a very strong feeling). The ability to use emotion to facilitate thought is indicated by matching various sensory experiences (color, temperature, speed) to emotions, whilst a series of multiple choice items testing knowledge of emotion definitions, transitions/blends, evaluates emotional understanding. For emotion management, participants are presented with a series of vignettes and asked to rate the usefulness of particular strategies for attaining a target feeling using a 5-point scale; 1 (not at all helpful) through to 5 (very helpful). Responses are scored in line with the degree of concordance with expert consensus opinion. Branch and total AEI scores can be derived (standardised values: $M=100, S D=15$ ) with higher scores indicating higher AEI skill. Acceptable split-half reliabilities have been obtained for the instrument (Papadogiannis et al., 2009), and the hierarchical four-factor structure has been recovered in data from youth aged 9-15 years, N=775 (Rivers et al., 2012). In the present sample, branch scores were significantly inter-correlated (average $r=.32$ ) and associated with the total score (range $r=.41$ [perceiving] - .81 [managing]).

The Emotional Quotient Inventory - Youth Version Short form (Bar-On \& Parker, 2000) is a 30-item self-report measure of TEI, responded to using a 4-point scale; 1 (not true of me: never, seldom) to 4 (very much true of me: very often). Summed item responses yield dimensional scores (range: 6-24) along with 'total EQ’ (range: 30-120), with higher scores reflecting higher emotional intelligence. See introduction for further details. In the present sample full scale $\alpha=.83$

The Trait Emotional Intelligence Questionnaire-Adolescent Short Form (Petrides, 2009) consists of 30 brief statements (e.g., "I find it hard to control my feelings”) which tap sociability, emotionality, self-control and well-being. Participants respond using a seven-point scale; 1 
PSYCHOMETRIC PROPERTIES: EQ-I:YVS

(strongly disagree) to 7 (strongly agree). Global TEI scores (possible range 30-210) are derived from summed item responses with higher scores signaling higher levels of TEI. The TEIQue has robust psychometric properties including 12-month test-retest reliability (Petrides, 2009) and full-scale internal consistency for the adolescent short form is typically in excess of $\alpha=.80$ (Mavroveli et al., 2007). In the present sample full scale $\alpha=.86$

The 20-item depression and disruptive behavior scales from the Beck Youth Inventories of Emotional and Social Impairment, Second edition (Beck, Beck, Jolly, \& Steer, 2005) indicated mental health. On each measure, participants specify how often each statement (e.g., “I feel lonely”; “I break into cars, houses or other places”; “I do things well”) has been true for them recently using a 4-point scale; 0 (never) through to 3 (always). Higher summed item values (range 0 - 60) represent higher levels of disorder. Normative standardization of the tool $(N=$ 1,000; aged 7-18 years), yielded excellent levels of internal consistency across three age bandings ( $\alpha \geq 0.86$ ) and 7-day test-retest reliability produced coefficients $\geq 0.73$ (Beck et al., 2005). In the current sample internal consistency was $\alpha=.93$ depression and $\alpha=.89$ disruptive behavior.

The Big Five Inventory-Adolescent Form (BFI-44-A; John, Donahue, \& Kentle, 1991) taps prototypical traits considered central to the 'Big Five' taxonomy of higher-order individual differences in Neuroticism (N); Extraversion (E); Openness (O); Agreeableness (A) and Conscientiousness (C). Participants indicate the extent of their agreement with 44 statements (e.g., "I see myself as someone who generates a lot of enthusiasm” [E]) using a five-point scale: 1 (strongly disagree) to 5 (strongly agree). Computation of item averages yields dimensional scores ( $n$ items per dimension range from 8-10). Soto, John, Gosling, \& Potter (2008) reported adequate levels of internal consistency and factorial validity in data drawn from 230,000 youth 
PSYCHOMETRIC PROPERTIES: EQ-I:YVS

aged 10-20 years old. In the present sample moderate alpha $(\alpha)$ values of .71(E); .62 (A); .75

$(\mathrm{C}) ; .70(\mathrm{~N}) ; .73(\mathrm{O})$ were recovered, which concurs with the younger age groups described in Soto et al., (2008).

\section{Results}

\section{Data screening}

The dataset showed some missing data for item responses, however, missing data

represented less than $1 \%$ of the overall dataset. Individual missing items were replaced with mean values as per guidance from individual measures. Where this was not possible, cases were removed pairwise, since Chi square analysis showed no significant association between missing cases and key demographic variables (assuming at least missing at random): sex; ethnicity (white British vs. other); free school meal eligibility. This resulted in a minimum sample between 720 and 849 (dependent on the analysis conducted), which comfortably exceeds recommended sample sizes for Confirmatory Factor Analysis (CFA) and additional analytical techniques used in this paper (Comrey \& Lee, 1992; Tabachnick \& Fidell, 2007). Mahalanobis distances did not identify any significant multivariate outliers and univariate normality was considered well within parameters - skew <.2.0 and kurtosis $<7.0$ (Curran, West \& Finch, 1996). Multicolinearity was not detected between domains (highest bivariate correlation $=.436$ ).

\section{Descriptive Statistics and Reliability}

Table 1 presents means, standard deviations and internal consistency coefficients (Cronbach's $\alpha$ ). Scores on most EQ-i:YV(S) subscales show moderate to high levels of internal consistency, with similar coefficients for both males and females. The intrapersonal scale is an exception, with a low alpha of .54 (.51 for females; .58 males). Males reported higher adaptability $(t(866)=3.03, p<.01 ; d=0.22)$, whilst females had higher interpersonal scores $(t$ 
PSYCHOMETRIC PROPERTIES: EQ-I:YVS

$(865)=7.98 p<.001, d=0.45)$, giving rise to a female advantage in total EQ scores $(t(847)=$ 2.29, $p<0.05, d=0.08)$. In considering age, bivariate correlations showed no significant effects for any of the EQ-i:YV(S) subscales, other than for intrapersonal scale (represented by a very small effect size: $\left.\mathrm{r}^{2}=-.129\right)$. This is consistent with previous examinations of developmental effects in EQi data, where scores were invariant across 12-18 year olds (Keefer et al., 2013). Table 2 shows the inter-item correlations of the EQ-i:YV(S) subscales. Subscales were moderately and positively related with the exception of stress management, where intercorrelations were weak or non-significant.

\section{Factor Structure}

Consistent with earlier validation studies (Parker, Keefer \& Wood, 2011), three CFA models were assessed: a one-factor model with all items loading into a single factor (model 1), a first order model with correlated latent variables for each of the four sub-domains of the EQi:YV(S) (model 2), and a second-order model examining the four sub domains indicated by a single latent factor (model 3). A range of model fit indicators were examined; root-mean-square error of approximation (RMSEA) (with 90\% confidence intervals), Standardised root-meansquare residual (SRMR), comparative fit index (CFI), and Tucker Lewis coefficient (TLI). Chisquare values were also examined with caution, given the tendency towards inflation of false positives in large samples (Hu \& Bentler, 1999). Model comparison was based on Akaike's information criterion (AIC) as a measure of comparative fit, where the lowest value represents the 'best fitting' model (Bozdogan, 2000). 'Ideal' fit indices draw from Hu and Bentler's recommendations (1999) and also from examination of indices reported for the tool elsewhere (Parker, Keefer \& Wood, 2011; Bar-On, 2004). See table 3 for results of the CFAs. 
PSYCHOMETRIC PROPERTIES: EQ-I:YVS

Model 1 provided a poor fit to the data, suggesting that response items from the EQi:YV(S) cannot be adequately explained by a single factor solution (i.e., total EQ). Consistent with this hypothesis is the improved fit indices for models 2 and 3, in which sub-domains of the EQ-i:YV(S) have been included. Model 2 (first order, correlated factors) showed that the latent domains of intrapersonal, interpersonal and adaptability were all moderately (.39 - .52) and significantly correlated, but stress management was orthogonal to the other domains, refuting its presence as a related construct. Model fit was not improved by including a higher-order, global EI factor (model 3). Although model 2 represented the best fit from the a-priori hypothesized models, it did not surpass ideal criteria provided by $\mathrm{Hu}$ and Bentler (1999), and further investigation was warranted. Individual factor loadings were all significant and within a range of $.44-.81$, with the exception of items 12 ('It is hard to talk about my deep feelings') and 26 ('I have trouble telling others about me feelings') which showed a negative correlation. Examination of standardised residuals suggested that model fit may be restricted by the underestimation of item correlations. This applied to items 28 and 30 from the interpersonal subscale ('I can tell when one of my close friends is unhappy'; 'I know when people are upset, even when they say nothing'), 12 and 26 from the intrapersonal subscale (as noted above), 5 and 27 from stress management ('I get too upset about things'; 'I get upset easily') and items 10 and 13 from the adaptability subscale ('I can understand hard questions'; I can come up with good answers to hard questions'). This intradimensional local dependence would appear to stem from similarity in wording (negative or positive endorsement) and content coverage which is common in measurement of personality constructs (Reise, Waller, \& Comrey, 2000) and has been found in previous EQi analyses with adult and adolescent short form data (Parker et al., 2011; Kun et al., 2012). These responses were allowed to correlate (Model 2 - corrected), resulting in an 
PSYCHOMETRIC PROPERTIES: EQ-I:YVS

improved model fit that satisfied ideal fit statistical criteria (table 3; figure 1 supplementary material).

\section{Measurement Invariance across Groups}

A multi-group CFA framework was used to assess measurement invariance across males and females. As recommended by Byrne (2012), initial baseline models (configural invariance models) were first calculated, followed by a test of invariant factor loadings (metric invariance) and invariant factor covariance (structural invariance). Measurement invariance is assumed true if the constrained models continue to fit the data well, in comparison to the baseline model (Byrne, 2012). Change in CFI was used to assess comparative fit across models, with a change of -.01 or less indicating equivalent fit (Vandenberg \& Lance, 2000), rather than $\mathrm{X}^{2}$, given the aforementioned difficulties with over-inflation. The configural invariance model $\mathrm{X}^{2}(440)=$ 1014.565, demonstrated an acceptable fit to the data (RMSEA $=.054,90 \%$ CI [.050, .059], CFI= .904) indicating that the subscales of the EQ-i:YV(S) were equivalent for males and females. The metric invariance model $X^{2}(459)=1039.855$, showed a similarly acceptable fit $(\mathrm{RMSEA}=$ $.054,90 \%$ CI $[.049, .058]$, CFI $=.903)$, indicating that scale items function in the same way for males and females $(\Delta \mathrm{CFI}=-.001)$. The structural invariance model $X^{2}(473)=1077.204$, also showed an acceptable fit (RMSEA $=.054,90 \%$ CI $[.050, .058]$, CFI $=.899)$ indicating that the there are no observable sex differences in either the latent EI dimensions or the correlations between them $(\triangle \mathrm{CFI}=-.004)$.

\section{Convergent and Discriminant Validity}

Consistent with Parker, Keefer, and Wood (2011), it was hypothesized that there should be a significant and meaningful bivariate correlation between the EQ-i:YV(S) and other measures of EI; TEIQue-ASF and MSCEIT:YV, yet correlations of lesser magnitude between 
PSYCHOMETRIC PROPERTIES: EQ-I:YVS

the EQ-i:YV(S) and the Big 5 personality dimensions (BFI-44-A sub-scales: Neuroticism; Extraversion; Openess; Conscientiousness; Agreeableness).

There was minimal compliance with the predicted hypotheses (see table 4). The EQiYV(S) shared small to moderate significant correlations with the TEIQue-ASF (.16 - .41), weak/negligible associations with the MSCEIT-YV(R) $(.02$ - .30) and a number of strong correlations with Big 5 personality dimensions e.g. Neuroticism and stress management; .48). However, correlational information constitutes a weak method for assessing construct validity since common method bias and uncontrolled measurement error can lead to spurious point estimates (Strauss \& Smith, 2009). In order to address this difficulty, a multitrait-multimethod latent-variable modeling approach (MTMM) (Campbell \& Fiske, 1959) was used to examine the convergent and divergent validity of the EQ-i:YV(S). This approach deconstructs error into its various components (i.e. trait, method, measurement), allowing for common method variance and measurement error to be controlled when estimating the associations between variables and a more accurate assessment of the construct validity of the EQ-i:YV(S).

For MTMM analysis, a matrix of methods and traits are required where one model (containing both traits [including their sub-domains] and method factors) is used as a reference standard to be compared against a number of further models in which traits (and their subdomains) or methods are constrained. Discrepancies in model fit can then be used to assess convergent and divergent validity. The EQ-i:YV(S), TEIQue-ASF, and MSCEIT:YV were considered to represent the same trait (EI), in contrast to the five factors of personality encapsulated by the BFI-44-A. Instruments were also distinguished by their method of assessment with the EQ-i:YV(S), TEIQue-ASF, and BFI-44-A utilizing self-report, and the MSCEIT:YV utilizing a maximal scoring method. Four models were constructed: 1) The 
PSYCHOMETRIC PROPERTIES: EQ-I:YVS

reference model in which both trait (EI and personality - including their subdomains) and method (self-report and maximal scoring) are specified; 2) a methods model in which only selfreport and maximal scoring are specified; 3) a trait model in which only EI and personality factors (including their subdomains) are specified; 4) a freely correlated model in which methods are not specified. Model fit was identified by significant change in chi-square $\left(\Delta \chi^{2}\right)$ and CFI ( $\triangle \mathrm{CFI}$ ) with a general recommendation that $\triangle \mathrm{CFI}$ should not exceed 0.010 (Cheung \& Rensvold, 2002). Results are shown in table 5.

Convergent validity is assessed by the extent to which independent measures of the same trait are correlated. This is done by comparing model 1 (in which traits are specified) with model 2 (in which they are not). For model 1, traits are defined as EI (as measured by the TEIQue-ASF and EQ-i:YV(S)) and personality factors (as measured by the BFI-44-A). $\chi^{2}$ provides a basis of judgment as to the extent of convergence, with a significant $\Delta \chi^{2}$ providing evidence for convergent validity. A significant difference in $\chi^{2}$ and CFI values $\left(\Delta \chi^{2} 575.617_{(18)}, p<.01\right)(\Delta \mathrm{CFI}$ $=0.172$ ) between models 1 and 2 provides evidence for convergent trait validity, as independent measures of the same trait are seen to be correlated. The fit statistics presented in table 5 imply that the EQi:YV(S) coheres with other measures of EI as anticipated.

Discriminant validity of traits can be assessed by comparing model 1 with model 3, in which independent measures of different traits (EI and personality factors as defined above) are correlated, as ideally these values should be negligible. Results indicate a significant difference between the models $\left(\Delta \chi^{2}-141.978_{(2)}, p<.01\right)$, consistent with expectations, however the change in practical fit $(\triangle \mathrm{CFI}=0.043$, past the 0.010 threshold indicated by Cheung \& Rensvold's threshold) is indicative of modest evidence for discriminant validity between EI and constructs of personality. 
PSYCHOMETRIC PROPERTIES: EQ-I:YVS

Method-based validity is assessed by comparing freely correlated factors (model 1) with uncorrelated models (model 4). Discriminant validity is supported by lack of model change (i.e., common method bias across self-report vs. ability measures). Significant model change was detected $\left(\Delta \chi 38.869_{(2)}, p<.01\right)$, and the comparatively small $\Delta \chi^{2}$ and $\Delta$ CFI $(0.011)$ suggests that evidence for the discriminant validity of the methods is stronger than that of trait. Although this indicates a sub-optimal fit with theory (as the correlation coefficients are more closely attributable to common method variance than discrimination between theoretical constructs, i.e. EI and personality traits), the data is broadly in line with recommendations originally noted by Campbell and Fisk (1959). A more probing consideration as to the extent to which this particular finding is problematic is hampered by a lack of more recent and contextualized benchmarks, which this article begins to address. In summary, there is modest evidence to support the validity of the EQ-i:YV(S) in relation to associated traits, however caution in interpretation is recommended to avoid conflating measurement and theoretical fit outside of a MTMM framework.

\section{Incremental and Predictive Validity}

Given known associations between personality and mental health outcomes (Malouff, Thorsteinsson, \& Schutte, 2005) it is important to assess whether the EQi:YV(S) can offer a unique contribution to the prediction of adolescent psychopathology. Two hierarchical regressions models were conducted. Depression and Disruptive Behavior served as the two criterion variables, with the Big Five dimensions entered in the first step, and total EQ-i:YV(S) scores entered in the second step. In step one, the Big Five traits explained $26.5 \%$ of observed variance in Depression $(F[5,827]=60.75, p<.001)$, and $22.7 \%$ of Disruptive Behavior $(F[5,825]=49.35, p<.001$. In step 2 (after controlling for the Big Five subscales), the EQ- 
PSYCHOMETRIC PROPERTIES: EQ-I:YVS

i:YV(S) total score did not account for a significant proportion of additional variance in Depression $(\Delta F(1,821)=1.512, p=.219)$ but explained an additional $1.7 \%$ in Disruptive Behavior $(\Delta F(1,819)=18.789, p<.001)$. Further analyses showed this effect could be attributed to the stress management subscale (see table 6). These findings are indicative of little useful incremental validity, given the small size of the correlation coefficients, once personality has been accounted for.

\section{Discussion}

The purpose of the current study was to be the first to provide a systematic psychometric evaluation of EQ-i:YV(S) data drawn from a large sample of UK adolescents. The multidimensional structure and brevity of the EQ-i:YV(S) make it an attractive option for measuring EI in time-limited settings (e.g., education; healthcare), where researchers seek to explore predictive outcomes in large samples of young people, or where rapid screening/profiling is desired. Limitations previously identified in analyses of the 'parent' instrument (EQ-i:YV long form) and derivative measures (EQ-i:YV brief) re-emerge in the current data, as well as some additional issues relating to predictive utility. These concerns will be discussed alongside practical implications for use of the measure.

\section{Factor Structure and Reliability}

The current study demonstrates modest reliability $(\alpha=.54-.84)$ in comparison to the original, normative data ( $\alpha=.72$ - .86; respondents aged 10-16 years) (Bar-On \& Parker, 2000). Only the adaptability subscale meets recommended levels of reliability for use in clinical work (.8-.9) (Smith, 2000), with estimates for the intrapersonal subscale significantly below even the more modest benchmarks set for EI instruments (.7) (Gignac, 2009). Inadequate reliabilities have also been reported in short-form data from Singaporean youth aged 13-15 years 
PSYCHOMETRIC PROPERTIES: EQ-I:YVS

(intrapersonal .62; interpersonal .64) (Wong \& Ang, 2007) and in 'parent’ EQ-i:YV long form data from UK adolescents (average $\alpha=$ intrapersonal .63) (Qualter et al., 2012). This is not unexpected given that the 6-item intrapersonal scale from the long form was retained in its entirety for the abridged short form. Findings from confirmatory factor analysis of the data shed some light on the difficulties inherent in this subscale.

A first-order model provided optimal fit to the data, suggesting EQ-i:YV(S) subscales are best represented as distinct, yet correlated variables as opposed to indicators of a single, global EI dimension. A higher-order model (global EI plus four subscales) also provided a favorable fit to the data, suggesting there is some shared variance at a global level (i.e., justifying 'total EQ'). However, two items belonging to the intrapersonal subscale (12: 'It is hard to talk about my deep feelings'; 26: 'I have trouble telling others about my feelings') were found to have very low factor loadings in the final model; far below the recommended cut-off of .45 for determining practical significance (Comrey \& Lee, 1992). These items were also poor discriminators of the intrapersonal dimension in analysis of long-form data from Canadian/American youth (Bar-On \& Parker, 2000; Parker et al., 2005), and were removed from structural analysis of Hungarian adolescent short-form data due to "lower statistical consistency and weaker association with the rest of the items” (Kun et al., 2012, p.522). Whilst all 6 intrapersonal scale items tap content relating to feelings (ability to describe; talk about feelings etc), items 12 and 26 differ in that they are reverse-keyed. It is possible that acquiescent responding (i.e., endorsing items irrespective of their content/wording) may account for this inconsistency, as this is a known feature of adolescent responding in personality data (Soto et al., 2008). Indeed, the stress management scale contains the only other reverse-keyed items included in the EQ-i:YV(S), and this dimension was found to be orthogonal to the other factors. Given these items tap an aspect of 
PSYCHOMETRIC PROPERTIES: EQ-I:YVS

emotion regulation (control of negative affect), which is a key component of all EI models (Petrides, 2011), this lack of coherency is unexpected on the grounds of content alone. Moreover, this does not appear to be a peculiarity of the current data having been similarly found in analyses of short and longitudinal, brief-form EQ-i data (Keefer et al., 2013; Kun et al., 2012). We propose, therefore, that there is some systematic error associated with two of the four EQi:YV(S) subscales and, until further short-form validation data become available (ideally crosscultural; longitudinal in design), researchers should limit their use of the instrument, particularly in high-stakes, clinical testing. The existing factor solution was, however, found to be equivalent across males and females (tested at configural, metric; structural levels), which indicates that the aforementioned difficulties are not a result of sex-related, differential item functioning.

\section{Construct Validity}

Correlational analyses demonstrated sub-optimal compliance with the predicted hypotheses. Specifically, there was a lower than expected degree of overlap between the EQ$\mathrm{i}$ YV(S) and both the TEIQue-ASF, and MSCEIT-YV(R), especially in comparison to Parker, Keefer and Wood (2011), although as Parker and colleagues analysis was based on adult EQi data, age-related developmental effects in EI may provide a partial explanation for this inconsistency (Parker, Saklofske, Wood, Eastabrook, \& Taylor, 2005). Once method bias and measurement error were accounted for through MTMM analyses, estimations were more consistent with predicted hypotheses. The EQi-YV(S) was shown to be congruent with other measures sharing the same trait (i.e. different measures of EI); however, there was still difficulty in establishing clear discriminant validity from personality, even after accounting for measurement bias. This issue has been noted in prior literature utilising similar analytical techniques. For instance, Joseph and Newman (2010) report up to 50\% shared variance between 
PSYCHOMETRIC PROPERTIES: EQ-I:YVS

trait Agreeableness, Conscientiousness, and EI. However establishing more precise benchmarks for adjudging 'adequate' discriminant validity is limited by a lack of MTMM analyses in the field.

As well as establishing convergent and divergent validity, measures of EI must also demonstrate their ability to predict adaptive outcomes over and above the influence of established (allied) constructs, to mitigate criticisms concerning conceptual redundancy (Shulte et al., 2004). Given the current drive to teach EI-related skills to young people to improve mental wellbeing (Durlak et al., 2011) and the conceptual overlap between TEI and broadband personality dimensions (Petrides, Pita, \& Kokkinaki, 2007), it is important to ascertain whether EQ-i:YV(S) scores offer something new in the prediction of key mental health criteria. This is particularly pertinent in light of the findings of the current MTMM analyses, but also as recent data suggest that only $38 \%$ of the variance in adult EQ-i scores may be 'novel' after accounting for significant contributions from trait Neuroticism, Extraversion, Conscientiousness and positive affect (Webb et al., 2013). As expected, we found that the Big Five dimensions accounted for a significant proportion of variance in adolescent mental health outcomes (Malouff et al., 2005), yet beyond the influence of these traits, total EQ-i:YV(S) scores were only able to predict unique variance in disruptive behavior (semi-partial $r=.150$ ). However, this small to medium effect (Cohen, 1992) was principally associated with stress management (semi-partial $r$ =.18), which, as noted above, showed a lack of coherency with the underlying model. Aside from Wong and Ang (2007), who also found this in EQ:i-YV(S) data predicting adolescent psychological maladjustment (antisocial behaviour; anger control; emotional distress), there are a lack of published sources against which to compare this finding. Normative data testing from the test developers did not extend to predictive validity for the EQ:i-YV(S). 
PSYCHOMETRIC PROPERTIES: EQ-I:YVS

There is now a good proportion of evidence to suggest that TEI measures should predict a range of mental health outcomes across the lifespan (Martins et al., 2010; Resurreccion, Salguero, \& Ruiz-Aranda, 2014) with unique capacity (e.g., Davis \& Humphrey, 2012), and this is central to the theoretical rationale behind the EQ:i model (Bar-On, 2004; Bar-On \& Parker, 2000). As earlier MTMM analyses showed a negligible proportion of overlap between measures due to shared method bias (i.e., associated with self-report) this suggests that there are difficulties with the content coverage of the EQ:i-YV(S). The veracity of the EQ-i model has been questioned over its differential sampling of the construct domain (Zeidner et al., 2009); whilst distinct 'EI' facets such as emotional understanding and regulation of emotion in others are omitted, peripherally-related personal capacities and qualities (e.g., problem-solving; social responsibility) are included. Consequently, the tool may be too broad to relate meaningfully to targeted indicators of adolescent mental health, such as depression and disruptive behavior, where difficulties in emotion regulation and emotional understanding/perception are known predictors (Mullin \& Hinshaw, 2007; Trentacosta \& Fine, 2010). The stress management subscale does, however, appear to measure a particular facet of emotion regulation (i.e., all 6 items tap poor control of negative affect). The problem here appears to be one of restricted content coverage and criterion contamination; rather than tapping traditional EI attributes relating to stress management (e.g., control of others' feelings to manage stressful situations; ability to cope with change), items are simply descriptors of negative emotional states which overlap with measures of mental health (e.g., EQ:i-YV(S): “I fight with people” vs. BYI II disruptive behavior: "I fight with others”). Thus, the association between self-perceived stress management skills and disruptive behavior becomes unreliable. Test developers should urgently review the content coverage of the tool and study relationships with a broader range of mental 
PSYCHOMETRIC PROPERTIES: EQ-I:YVS

health indicators to ascertain the scale of this issue. It may be that the EQ:i-YV(S) is appropriate for predicting other types of adaptation in young people (e.g., educationally relevant outcomes, Perera \& DiGiacomo, 2013) and its use could be restricted to this arena. In its current form, the authors advise caution when interpreting EQ:i-YV(S) sub-scale scores, particularly alongside measures of internalizing and externalizing disorder. Other short form measures of TEI suitable for use with young people may be more appropriate such as the TEIQue-ASF or newer, multidimensional measures such as the Emotional Self-Efficacy Scale for Youth (Qualter et al., 2014). For instance, the suite of TEIQue questionnaires include items tapping 'stress management', ‘emotional regulation', 'impulsivity’ and 'adaptability’ under the broader rubric of 'self-control', derived from content analysis of leading EI models and related constructs (e.g., alexithymia) (Petrides, 2011).

\section{Conclusions}

A rather mixed picture emerges from this first systematic psychometric examination of the EQ:i-YV(S). Despite confirming a multidimensional, sex-equivalent structure for the tool, sub-optimal convergent and divergent validity and serious underlying issues concerning the reliability, structural coherency, and predictive validity were revealed. As a result, the current authors recommend that researchers avoid using the EQ:i-YV(S) until further validation data are collected and the functioning of scale items can be definitively established in relation to a wider range of predictive outcomes. This is especially relevant to researchers seeking to use the scale for predictive or evaluative purposes in clinical settings. It has been suggested that content coverage and item wording could be central difficulties, which ultimately impact the integrity and use of the tool in its current form. These appear to be 'carry-over' effects from the youth long form measure from which the tool was derived, where there was limited normative testing 
PSYCHOMETRIC PROPERTIES: EQ-I:YVS

using a single sample of data - a practice cautioned against in the literature relating to short-form development (Smith, 2000). These issues do not appear to be culturally-specific, as similar findings have been reported elsewhere in other UK; Hungarian; Canadian; American and Singaporean data sets (Keefer et al., 2013; Kun et al., 2012; Parker et al., 2005; Qualter et al., 2012). However this should be definitively confirmed using alternative analytical methods such as differential item functioning to compare cross-cultural responses (Ekermans, Saklofske, Austin, \& Stough, 2011). Whilst this is the only study to test competing structural models of EQ:i-YV(S) data via CFA, researchers should also seek to collect (or pool data) to allow for cross-validation of structural models. 
PSYCHOMETRIC PROPERTIES: EQ-I:YVS

\section{References}

Bar-On, R. (2004). The Bar-On Emotional Quotient Inventory (EQ-i): Rationale, description and summary of psychometric properties. In G. Geher (Ed.), Measuring Emotional Intelligence: Commen Ground and Controversy (pp. 111142). Hauppauge, NY: Nova Science.

Bar-On, R., \& Parker, J. D. (2000). BarOn Emotional Quotient Inventory: Youth Version - Technical Manual. Toronto, Canada: Multi-Health Systems Inc.

Beck, J. S., Beck, A. T., Jolly, J. B., \& Steer, R. A. (2005). Manual for the Beck Youth Inventories of Emotional and Social Impairment (2nd ed.). San Antonio, TX: Psychological Corporation.

Brackett, M., Rivers, S. E., Reyes, M. R., \& Salovey, P. (2012). Enhancing academic performance and social and emotional competence with the RULER feeling words curriculum. Learning and Individual Differences, 22(2), 218-224.

Brackett, M., Rivers, S. E., \& Salovey, P. (2011). Emotional Intelligence: Implications for Personal, Social, Academic, and Workplace Success. Social and Personality Psychology Compass, 5(1), 88-103.

Brody, N. (2004). What cognitive intelligence is and what emotional intelligence is not. Psychological Inquiry, 15(3), 234-238.

Campbell, D., \& Fiske, D. (1959). Convergent and discriminant validation by the multi-trait multi-method matrix. Psychological Bulletin, 56(2), 81-105.

Cheung, G. W., \& Rensvold, R. B. (2002). Evaluating goodness-of-fit indexes for testing measurement invariance. Structural equation modeling, 9(2), 233255. 
PSYCHOMETRIC PROPERTIES: EQ-I:YVS

Cohen, J. (1992). A Power Primer. Psychological Bulletin, 112(1), 155-159.

Comrey, A. L., \& Lee, H. B. (1992). A first course in factor analysis (2nd ed.). Hillsdale, NJ: Lawrence Erlbaum Associates.

Davis, S. K., \& Humphrey, N. (2012). Emotional intelligence predicts adolescent mental health beyond personality and cognitive ability. Personality and Individual Differences, 52(2), 144-149.

Davis, S. K., \& Humphrey, N. (2014). Ability versus trait emotional intelligence: Dual influences on adolescent psychological adaptation. Journal of Individual Differences, 35(35), 54-62.

Department for Education. (2011). Statistical First Release: Schools, Pupils and their Characteristics, June 2011. Retrieved from http://www.education.gov.uk/rsgateway/DB/SFR/s001012/index.shtml

Durlak, J. A., Weissberg, R. P., Dymnicki, A. B., Taylor, R. D., \& Schellinger, K. B. (2011). The Impact of Enhancing Students' Social and Emotional Learning: A Meta-Analysis of School-Based Universal Interventions. Child development, 82(1), 405-432.

Ekermans, G., Saklofske, D. H., Austin, E., \& Stough, C. (2011). Measurement invariance and differential item functioning of the Bar-On EQ-i: S measure over Canadian, Scottish, South African and Australian samples. Personality and Individual Differences, 50(2), 286-290.

Gignac, G. E. (2009). Psychometrics and the measurement of Emotional Intelligence. In C. Stough, D. H. Saklofske \& J. D. A. Parker (Eds.), Assessing Emotional 
PSYCHOMETRIC PROPERTIES: EQ-I:YVS

Intelligence: Theory, Research and Applications (pp. 9-40). New York: Springer.

Humphrey, N., Kalambouka, A., Wigelsworth, M., Lendrum, A., Deighton, J., \& Wolpert, M. (2011). Measures of Social and Emotional Skills for Children and Young People. Educational and Psychological Measurement, 71(4), 617-637.

John, O. P., Donahue, E. M., \& Kentle, R. L. (1991). The Big Five Inventory: Versions $4 a$ and 54. Berkeley, CA: University of California, Berkeley, Institute of Personality and Social Research.

Joseph, D. L., \& Newman, D. A. (2010). Discriminant Validity of Self-Reported Emotional Intelligence: A Multitrait-Multisource Study. Educational and Psychological Measurement, 70(4), 672-694.

Keefer, K. V., Holden, R. R., \& Parker, J. D. (2013). Longitudinal assessment of trait emotional intelligence: Measurement invariance and construct continuity from late childhood to adolescence. Psychological Assessment, 25(4), 12551272.

Kristensen, H. A., Parker, J. D., Taylor, R. N., Keefer, K. V., Kloosterman, P. H., \& Summerfeldt, L. J. (2014). The relationship between trait emotional intelligence and ADHD symptoms in adolescents and young adults. Personality and Individual Differences, 65(0), 36-41.

Kun, B., Urban, R., Paksi, B., Csobor, L. V., Olah, A., \& Demetrovics, Z. (2012). Psychometric characteristics of the Emotional Quotient Inventory, Youth Version, Short Form, in Hungarian high school students. Psychological Assessment, 24(2), 518-523. 
PSYCHOMETRIC PROPERTIES: EQ-I:YVS

MacCann, C., Wang, L., Matthews, G., \& Roberts, R. D. (2010). Emotional intelligence and the eye of the beholder: Comparing self- and parent-rated situational judgments in adolescents. Journal of Research in Personality, 44(5), 673-676.

Malouff, J. M., Thorsteinsson, E. B., \& Schutte, N. S. (2005). The Relationship Between the Five-Factor Model of Personality and Symptoms of Clinical Disorders: A Meta-Analysis. Journal of Psychopathology and Behavioral Assessment, 27(2), 101-114.

Martins, A., Ramalho, N., \& Morin, E. (2010). A comprehensive meta-analysis of the relationship between Emotional Intelligence and health. Personality and Individual Differences, 49(6), 554-564.

Mavroveli, S., Petrides, K. V., Rieffe, C., \& Bakker, F. (2007). Trait emotional intelligence, psychological well-being and peer-rated social competence in adolescence. British Journal of Developmental Psychology, 25(2), 263-275.

Mavroveli, S., \& Sánchez-Ruiz, M. J. (2011). Trait emotional intelligence influences on academic achievement and school behaviour. British Journal of Educational Psychology, 81(1), 112-134.

Mayer, J. D., Roberts, R. D., \& Barsade, S. G. (2008). Human abilities: Emotional intelligence. Annual Review of Psychology, 59, 507-536.

Mayer, J. D., Salovey, P., \& Caruso, D. R. (in press). Mayer-Salovey-Caruso Emotional Intelligence Test: Youth Version - Research Edition (MSCEIT-YV). Toronto: Multi-Health Systems. 
PSYCHOMETRIC PROPERTIES: EQ-I:YVS

Mullin, B. C., \& Hinshaw, S. P. (2007). Emotion regulation and externalizing disorders in children and adolescents. In J. J. Gross (Ed.), Handbook of emotion regulation (pp. 523-541). New York: Guildford Press.

Papadogiannis, P. K., Logan, D., \& Sitarenios, G. (2009). An ability model of emotional intelligence: A rationale, description, and application of the Mayer Salovey Caruso Emotional Intelligence Test (MSCEIT). In C. Stough, D. H. Saklofske \& J. D. A. Parker (Eds.), Assessing emotional intelligence: Theory, research and application (pp. 43-65). New York: Springer.

Parker, J. D., Keefer, K. V., \& Wood, L. M. (2011). Toward a Brief Multidimensional Assessment of Emotional Intelligence: Psychometric Properties of the Emotional Quotient Inventory-Short Form. Psychological Assessment, 23(3), 762-777.

Parker, J. D., Saklofske, D. H., Shaughnessy, P. A., Huang, S. H. S., Wood, L. M., \& Eastabrook, J. M. (2005). Generalizability of the emotional intelligence construct: A cross-cultural study of North American aboriginal youth. Personality and Individual Differences, 39(1), 215-227.

Parker, J. D., Saklofske, D. H., Wood, L. M., Eastabrook, J. M., \& Taylor, R. N. (2005). Stability and change in emotional intelligence: Exploring the transition to young adulthood. Journal of Individual Differences, 26(2), 100-106.

Parker, J. D., Summerfeldt, L. J., Taylor, R. N., Kloosterman, P. H., \& Keefer, K. V. (2013). Problem gambling, gaming and Internet use in adolescents: Relationships with emotional intelligence in clinical and special needs samples. Personality and Individual Differences, 55(3), 288-293. 
PSYCHOMETRIC PROPERTIES: EQ-I:YVS

Perera, H. N., \& DiGiacomo, M. (2013). The relationship of trait emotional intelligence with academic performance: A meta-analytic review. Learning and Individual Differences, 28, 20-33.

Perez, J. C., Petrides, K. V., \& Furnham, A. (2005). Measuring trait emotional intelligence. In R. Schultz \& R. D. Roberts (Eds.), International handbook of emotional intelligence. (pp. 123-143). Cambridge, MA: Hogrefe \& Huber.

Petrides, K. V. (2009). Technical manual for the trait emotional intelligence questionnaires (TEIQue) (1st ed.). London: London Psychometric Laboratory.

Petrides, K. V. (2011). Ability and Trait Emotional Intelligence. In T. ChamorroPremuzic, S. von Stumm \& A. Furnham (Eds.), The Wiley-Blackwell Handbook of Individual Differences (pp. 656-679). UK: Blackwell Publishing.

Petrides, K. V., Pita, R., \& Kokkinaki, F. (2007). The location of trait emotional intelligence in personality factor space. British Journal of Psychology, 98(2), 273-289.

Qualter, P., Gardner, K. J., Pope, D. J., Hutchinson, J. M., \& Whiteley, H. E. (2012). Ability emotional intelligence, trait emotional intelligence, and academic success in British secondary schools: A 5-year longitudinal study. Learning and Individual Differences, 22(1), 83-91.

Qualter, P., Pool, L. D., Gardner, K. J., Ashley-Kot, S., Wise, A., \& Wols, A. (2014). The Emotional Self-Efficacy Scale: Adaptation and Validation for Young Adolescents. Journal of Psychoeducational Assessment.

Reise, S. P., Waller, N. G., \& Comrey, A. L. (2000). Factor analysis and scale revision. Psychological Assessment, 12, 287-297. 
PSYCHOMETRIC PROPERTIES: EQ-I:YVS

Resurreccion, D. M., Salguero, J. M., \& Ruiz-Aranda, D. (2014). Emotional intelligence and psychological maladjustment in adolescence: A systematic review. Journal of Adolescence, 37(4), 461-472.

Rivers, S. E., Brackett, M., Reyes, M. R., Mayer, J. D., Caruso, D. R., \& Salovey, P. (2012). Measuring Emotional Intelligence in Early Adolescence With the MSCEIT-YV. Journal of Psychoeducational Assessment, 30(4), 344-366.

Saklofske, D. H., Austin, E. J., \& Minski, P. S. (2003). Factor structure and validity of a trait emotional intelligence measure. Personality and Individual Differences, $34(4), 707-721$.

Schulte, M. J., Ree, M. J., \& Carretta, T. R. (2004). Emotional intelligence: not much more than g and personality. Personality and Individual Differences, 37(5), 1059-1068.

Schutte, N. S., Malouff, J. M., Hall, L. E., Haggerty, D. J., Cooper, J. T., Golden, C. J., \& Dornheim, L. (1998). Development and validation of a measure of emotional intelligence. Personality and Individual Differences, 25(2), 167-177.

Smith, G. T., McCarthy, D. M., \& Anderson, K. G. (2000). On the sins of short-form development. Psychological Assessment, 12(1), 102-111.

Soto, C. J., John, O. P., Gosling, S. D., \& Potter, J. (2008). The developmental psychometrics of big five self-reports: Acquiescence, factor structure, coherence, and differentiation from ages 10 to 20. Journal of Personality and Social Psychology, 94(4), 718-737.

Strauss, M. E., \& Smith, G. T. (2009). Construct validity: Advances in theory and methodology. Annual Review of Clinical Psychology, 5(1), 1-25. 
PSYCHOMETRIC PROPERTIES: EQ-I:YVS

Trentacosta, C. J., \& Fine, S. E. (2010). Emotion Knowledge, Social Competence, and Behavior Problems in Childhood and Adolescence: A Meta-analytic Review. Social Development, 19(1), 1-29.

Van Rooy, D. L., \& Viswesvaran, C. (2004). Emotional intelligence: A meta-analytic investigation of predictive validity and nomological net. Journal of Vocational Behavior, 65(1), 71-95.

Webb, C. A., Schwab, Z. J., Weber, M., DelDonno, S., Kipman, M., Weiner, M. R., \& Killgore, W. D. S. (2013). Convergent and divergent validity of integrative versus mixed model measures of emotional intelligence. Intelligence, 41(3), 149-156.

Wilhelm, O. (2005). Measures of emotional intelligence: Practice and standards. In R. Schulze \& R. Roberts (Eds.), International Handbook of Emotional Intelligence (pp. 131-154). Seattle, WA: Hogrefe \& Huber.

Williams, C., Daley, D., Burnside, E., \& Hammond-Rowley, S. (2009). Measuring emotional intelligence in preadolescence. Personality and Individual Differences, 47(4), 316-320.

Wong, S. S., \& Ang, R. P. (2007). Emotional competencies and maladjustment in Singaporean adolescents. Personality and Individual Differences, 43(8), 21932204.

Zeidner, M., Matthews, G., \& Roberts, R. D. (2009). What we know about emotional intelligence: How it affects learning, work, relationships, and our mental health. Cambridge, MA: MIT Press. 
Table 1. Whole sample and sex-specific means, standard deviations and alpha coefficients from the EQ-i:YV(S) subscales

\begin{tabular}{|c|c|c|c|c|c|c|c|c|c|c|}
\hline \multirow[b]{2}{*}{ Subscale } & \multicolumn{3}{|c|}{$\begin{array}{c}\text { Total sample }(\mathrm{N}= \\
849)\end{array}$} & \multicolumn{3}{|c|}{ Males $(\mathrm{N}=442)$} & \multicolumn{3}{|c|}{ Females $(\mathrm{N}=407)$} & \multirow[b]{2}{*}{$t$} \\
\hline & Mean & $S D$ & Alpha & Mean & $S D$ & Alpha & Mean & $S D$ & Alpha & \\
\hline Interpersonal & 17.87 & 3.56 & .77 & 16.98 & 3.60 & .76 & 18.53 & 3.24 & .75 & $7.98^{* * *}$ \\
\hline Intrapersonal & 13.80 & 3.18 & .54 & 13.42 & 3.20 & .58 & 13.72 & 3.20 & .51 & 0.83 \\
\hline Adaptability & 15.80 & 3.92 & .84 & 16.22 & 3.93 & .84 & 15.34 & 3.87 & .84 & $3.03^{*}$ \\
\hline $\begin{array}{l}\text { Stress } \\
\text { management }\end{array}$ & 12.84 & 12.84 & .71 & 12.69 & 3.94 & .76 & 13.00 & 4.02 & .75 & 1.13 \\
\hline Total EQ & 60.32 & 9.36 & .80 & 59.61 & 9.82 & .85 & 61.08 & 8.78 & .80 & $2.29 *$ \\
\hline
\end{tabular}

Table 2. Inter-item correlations including age effects

\begin{tabular}{|c|c|c|c|c|c|}
\hline & Interpersonal & Intrapersonal & Adaptability & $\begin{array}{l}\text { Stress } \\
\text { management }\end{array}$ & Age $\left(r^{2}\right)$ \\
\hline Interpersonal & - & & & & .04 \\
\hline Intrapersonal & $.34 * *$ & - & & & $-.13 * *$ \\
\hline Adaptability & $.44^{* *}$ & $.38 * *$ & - & & -.02 \\
\hline $\begin{array}{l}\text { Stress } \\
\text { management }\end{array}$ & .10 & $.15^{* *}$ & -.05 & - & .02 \\
\hline
\end{tabular}

$* * p<.01$

Table 3. CFA fit indices $(\mathrm{N}=720)$

\begin{tabular}{lcccccccc}
\hline \multicolumn{1}{c}{ Model } & $\mathrm{X}^{2}$ & $\mathrm{df}$ & $\mathrm{RMSEA}$ & $90 \%$ CI & SRMR & CFI & TLI & AIC \\
\hline 'ideal' & - & - & $\approx 0$ & - & $\approx 0$ & $>.9$ & $>.9$ & - \\
\hline Model 1 & $3616.141^{*}$ & 252 & .125 & {$[.121, .129]$} & .118 & .460 & .409 & 53543.651 \\
Model 2 & $1252.766^{*}$ & 246 & .068 & {$[.064, .072]$} & .068 & .843 & .8241 & 51092.276 \\
Model 3 & $1259.814^{*}$ & 248 & .068 & {$[.064, .072]$} & .069 & .842 & .825 & 51095.324 \\
\hline $\begin{array}{l}\text { Model 2 } \\
\text { (corrected) }\end{array}$ & $\mathbf{7 3 1 . 9 8 5 *}$ & $\mathbf{2 2 0}$ & $\mathbf{. 0 5 1}$ & {$[\mathbf{. 0 4 7}, \mathbf{. 0 5 6}]$} & $\mathbf{. 0 6 0}$ & $\mathbf{. 9 1 4}$ & $\mathbf{. 9 0 1}$ & $\mathbf{4 8 8 3 6 . 6 0 1}$ \\
\hline
\end{tabular}

Note. Model 1 = one-factor model; Model 2 = correlated first order model; model $3=$ second-order model; $\mathrm{X}^{2}=$ chi-square test statistic; $\mathrm{df}=$ Degrees of freedom; RMSEA = root-mean square error of approximation; $90 \% \mathrm{CI}$

$=90 \%$ Confidence interval for RMSEA; SRMR $=$ Standardised root-mean-square residual; CFI $=$ Comparative fit index; TLI = Tucker-Lewis index; AIC = Akaike Information Criterion.

$* \mathrm{p}<.001$ 
Table 4. Bivariate correlations between the EQ-i:YV(S) and validation measures

\begin{tabular}{|c|c|c|c|c|c|}
\hline Variable & Interpersonal & Intrapersonal & Adaptation & Stress & Total EQ \\
\hline \multicolumn{6}{|l|}{ TEIQue-ASF } \\
\hline Total TEI & $.16^{* *}$ & $.26^{* *}$ & $.38 * *$ & $-.41 * *$ & $.16^{* *}$ \\
\hline \multicolumn{6}{|l|}{ MSCEIT-YV (R) } \\
\hline Perceiving emotions & -.03 & .07 & .05 & $.09 *$ & $.08 *$ \\
\hline Facilitating emotions & $-26 * *$ & $.08 *$ & $.16^{* *}$ & .05 & $.21 * *$ \\
\hline Understanding emotions & $.26 * *$ & $-.10 * *$ & $.14^{* *}$ & $-.10 * *$ & .07 \\
\hline Managing emotions & $.30 * *$ & -.01 & $.12 * *$ & .07 & $.12^{* *}$ \\
\hline Total AEI & -.02 & -.04 & $.15^{* *}$ & $-.07 *$ & $.13^{* *}$ \\
\hline \multicolumn{6}{|l|}{ Big 5} \\
\hline Neuroticism & .00 & $-.15 * *$ & $-.26 * *$ & $.48 * *$ & .06 \\
\hline Extraversion & $.18^{* *}$ & $.19 * *$ & $.25^{* *}$ & $-.08 *$ & $.21^{* *}$ \\
\hline Openness & $.36 * *$ & $.11^{* *}$ & $.36^{* *}$ & -.02 & $.32 * *$ \\
\hline Conscientiousness & $.27 * *$ & $.20 * *$ & $.37 * *$ & $-.31 * *$ & $.20 * *$ \\
\hline
\end{tabular}

TEI = Trait emotional intelligence; AEI = ability emotional intelligence

${ }^{*} \mathrm{p}<.05,{ }^{* *} \mathrm{p}<.01$

Table 5. Summary of MTMM latent variable models

\begin{tabular}{|c|c|c|c|c|c|c|}
\hline Model & $\chi^{2}$ & $d f$ & CFI & RMSEA & $\begin{array}{l}\text { RMESA } \\
90 \% \text { CI }\end{array}$ & SRMR \\
\hline $\begin{array}{l}\text { 1.Freely correlated traits, freely } \\
\text { correlated methods }\end{array}$ & 720.566 & 100 & 0.809 & 0.093 & $\begin{array}{l}0.087 \\
0.100\end{array}$ & 0.062 \\
\hline $\begin{array}{l}\text { 2. Trait-free, freely correlated } \\
\text { methods }\end{array}$ & 1296.183 & 118 & 0.637 & 0.119 & $\begin{array}{l}0.113 \\
0.125\end{array}$ & 0.100 \\
\hline $\begin{array}{l}\text { 3.Perfectly correlated traits*, freely } \\
\text { correlated methods }\end{array}$ & 862.544 & 102 & 0.766 & 0.103 & $\begin{array}{l}0.096 \\
0.109\end{array}$ & 0.084 \\
\hline $\begin{array}{l}\text { 4.Freely correlated traits*, method- } \\
\text { free }\end{array}$ & 759.425 & 102 & 0.798 & 0.095 & $\begin{array}{l}0.089 \\
0.102\end{array}$ & 0.068 \\
\hline
\end{tabular}

Note. Traits = EI (EQi-YV(s), MSCEIT:YV, TEIQue-ASF), Personality (BFI-44-A); Methods = Self report

(EQi-YV(s), TEIQue-ASF, BFI-44-A), maximal scoring (MSCEIT:YV). $\mathrm{X}^{2}=$ chi-square test statistic; df =

Degrees of freedom; CFI = Comparative fit index; RMSEA = root-mean square error of approximation; 90\% CI

= 90\% Confidence interval for RMSEA; SRMR = Standardised root-mean-square residual

*Represents re-specified model with residual (BFIO fixed at 0.0) 
Table 6. Summary of hierarchical regression analyses for prediction of depression and disruptive behavior.

\begin{tabular}{lcccc}
\hline Predictors & Adjusted $R^{2}$ & $\beta$ & Semi-partial $r$ & $p$ \\
\hline Depression & & & & \\
$\quad$ Step 1 & .265 & & & \\
$\quad$ Extroversion & & -.116 & -.121 & $<.001$ \\
$\quad$ Agreeableness & & -.041 & -.040 & .250 \\
$\quad$ Conscientiousness & & -.075 & -.074 & .034 \\
$\quad$ Neuroticism & & .429 & .400 & $<.001$ \\
$\quad$ Openness & & .105 & .110 & .002 \\
Step 2 & .266 & & & \\
EQI Total & & .040 & .055 & .219 \\
Disruptive Behaviour & & & & \\
Step 1 & .227 & & & \\
$\quad$ Extroversion & & -.014 & -.014 & .690 \\
Agreeableness & & -.409 & -.360 & $<.001$ \\
Conscientiousness & & -.122 & -.116 & .001 \\
$\quad$ Neuroticism & & .015 & .015 & .669 \\
$\quad$ Openness & & .032 & .033 & .348 \\
Step 2 & .243 & & & \\
$\quad$ EQI Total & & .142 & .150 & $<.001$ \\
Step 2 & .259 & & & \\
Interpersonal & & -.053 & -.049 & .166 \\
Intrapersonal & & .033 & .034 & .338 \\
Adaptability & & .061 & .057 & .102 \\
Stress Management & & .202 & .184 & $<.001$ \\
\hline
\end{tabular}

